\title{
Range verification by means of prompt-gamma detection in particle therapy ${ }^{1}$
}

\author{
Aleksandra Wrońska \\ Marian Smoluchowski Institute of Physics, Jagiellonian University, Łojasiewicza 11, \\ 30-348 Kraków, Poland \\ E-mail: aleksandra.wronska@uj.edu.pl \\ Denis Dauvergne \\ Université Grenoble Alpes, CNRS/IN2P3, Grenoble INP, LPSC-UMR 5821, 38000 Grenoble, \\ France \\ E-mail: denis.dauvergne@lpsc.in2p3.fr
}

\begin{abstract}
Ion range verification in particle therapy, possibly in real time, is still an open issue to improve the treatment quality by reducing safety margins. Among the various methods that are under study or already brought to the clinics, prompt gamma offer a unique opportunity of real time control. In this chapter, we present the physical background and conditions specific for the operation environment in the clinics, followed by an overview of the various techniques relying on prompt-gamma detection that have been proposed and developed for more than a decade. Different approaches, based on imaging and non-imaging techniques, providing either integrated or differential information are described, and their maturity, limitations and clinical usefulness are discussed.
\end{abstract}

\section{Introduction}

Over seventy years ago Robert Wilson proposed to use ion beams for tumour treatment [2]. He combined the theoretical developments of Hans Bethe describing energy losses of protons and heavier ions traversing a medium, with the technical achievements of Ernest O. Lawrence, who built the first cyclotron. Currently, ion beam therapy (known also as particle therapy) is among most important methods of tumour treatment, next to surgery, chemotherapy, conventional radiotherapy and emerging immunotherapy (Nobel prize in medicine 2018).

Even though heavier ions exhibit larger biological effectiveness of the deposited dose and allow better dose conformation to the tumour volume, their use is strongly limited by much larger construction and maintenance costs as well as technological complexity of the treatment sites. Consequently, protons remain the main type of ions used for ion beam radiotherapy, as proposed in Wilson's original paper.

The field of particle therapy (PT) was rapidly developing over those seven decades: from the knowledge about how to fully exploit the potential of the Bragg peak, through the benefits

1 The chapter has been written based on the ref. [1]. The figures have been reprinted/adapted from there as well, unless stated otherwise. 
of fractionated delivery to stretching the region of maximum dose (spread-out Bragg peak). In the 1990s there was a transfer of technology from research centres to hospitals. About the same time, commercial companies entered the game and around 2000 already the off-the-shelf solutions for ion beam therapy became available. The XXI century has brought further progress: multi-field irradiation, the use of gantries and scanning pencil beams, modern CT- and PETassisted evaluation of treatment plans based on sophisticated computer simulations [3]. Those developments made the field flourish, the number of ion-beam therapy centres grew rapidly and is currently close to 100, with another 40 under construction [4].

Particle therapy takes advantage of the unique features of ion interaction with matter with its characteristic Bragg peak, allowing to almost arbitrarily shape the distribution of deposited dose. The well-defined ion range in the medium leads to a rapid falloff to zero in dose depth profile, allowing to spare deeper located tissues. However, the existence of the Bragg peak and its steep distal falloff in the dose depth profile which are the main advantages of charged heavy particles over X-rays make the proton and ion therapy more susceptible to errors. A detailed quantitative analysis of sources of proton beam range uncertainties, such as patient positioning, patient anatomical changes and translation of CT images to water equivalent units, was performed by Paganetti [5]. In clinical practice, this leads to the necessity of using safety margins, i.e. enlarging the irradiated volume. The values of those margins defined differently in each PT centre, typical values vary from a few millimetres up to over a centimetre for deeply located malignancies. They are necessary to account for possible uncontrolled variations of beam range within uncertainties, but they also mean an extra dose delivered to patient's healthy tissues. The way to reduce beam range uncertainties and consequently safety margins would mean a lower dose delivered to a patient and thus lower risk of long-term side effects. This can be achieved by on-line monitoring of the deposited dose distribution during treatments [6-8]. The development of necessary tools was one of the highlights of the 2014 NuPECC report [9].

There are different approaches to ion beam therapy monitoring currently under development. Most of them are based on the idea of exploiting by-products of patient irradiation with proton beam: prompt gamma radiation, which is the main focus of this chapter, but also acoustic wave $[10 ; 11]$, secondary protons during carbon therapy [12], secondary electron bremsstrahlung [13], neutrons [14] and $\beta^{+}$-emitters [15]. For the latter, the range verification consists in imaging the decay of the $\beta^{+}$-emitters in two photons, by means of a Positron Emission Tomography (PET) device. In this domain, spectacular progress was seen in the last years. Until recently, PET scans were considered useful for post-irradiation control rather than in vivo range verification [16] due to long acquisition times necessary to collect sufficient statistics resulting from limited acceptance of PET scanners and long lifetimes of $\beta^{+}$emitters. This, in turn, opened a way to biological washout which blurred the image. Dendooven et al. proposed to eliminate this obstacle by focusing on short-lived $\beta^{+}$-emitters [17] and tested the experimental feasibility of such a solution [18]. A real breakthrough, however, came with the development of a high-acceptance, high-efficiency INSIDE setup currently operated at CNAO, Pavia [19]. In fact, this setup combines in-beam PET with secondary particle tracking. The first clinical test with a patient was reported in 2018 [20]. Range agreement within $1 \mathrm{~mm}$ was demonstrated, which proved the method and setup useful for verification of dose distribution in proton and carbon therapy. The issue of compliance of in-beam PET with the delivery mode remains, which necessitates either dual head (like for the former GSI prototype and INSIDE), or specific geometries like the dual ring Open-PET developed at NIRS [21]. In-beam PET records signal during beam pauses, which makes it suitable with low duty-cycle accelerators, otherwise inroom or offline PET imaging can be performed with commercial PET-CT, at the expense of the washout effect and long acquisition times. Note also that ongoing developments on ToF-PET would be beneficial towards real-time imaging to reduce background. Lastly, PET presents an additional asset in the case of carbon/oxygen therapy, since projectile-like $\beta^{+}$emitters decay 
close to the Bragg peak location, giving rise to enhanced signal.

Of the listed secondary radiations, prompt gamma radiation (PG), typically of a few $\mathrm{MeV}$ energy (from 1-2 MeV up to about $7 \mathrm{MeV}$ ), has the advantage that it is produced instantaneously (ps time scale), and penetrates the tissue easily, mostly without any interaction on its way out. Thus, the information about the location of its origin is not distorted. The characteristics of the PG radiation allows to pinpoint the Bragg peak position [22-24]. Fig. 1 illustrates the correlation between the deposited-dose depth-profile and the various secondary-particle vertex distributions caused by inelastic collisions in the case of proton beams. In the present case, the vertex distributions correspond to the particles with energy above $1 \mathrm{MeV}$ emerging from a water cylinder of $15 \mathrm{~cm}$ diameter and $40 \mathrm{~cm}$ length, irradiated by $160 \mathrm{MeV}$ protons (Geant4 simulations, adapted from [25]). A clear correlation between PG vertices and dose is observed, although part of PGs are emitted by secondary particles (mostly neutrons). Note, that the neutron vertex distribution is also well correlated to the projectile range, but, for the latter, the information on the vertex location will be blurred by scattering in the phantom before detection.

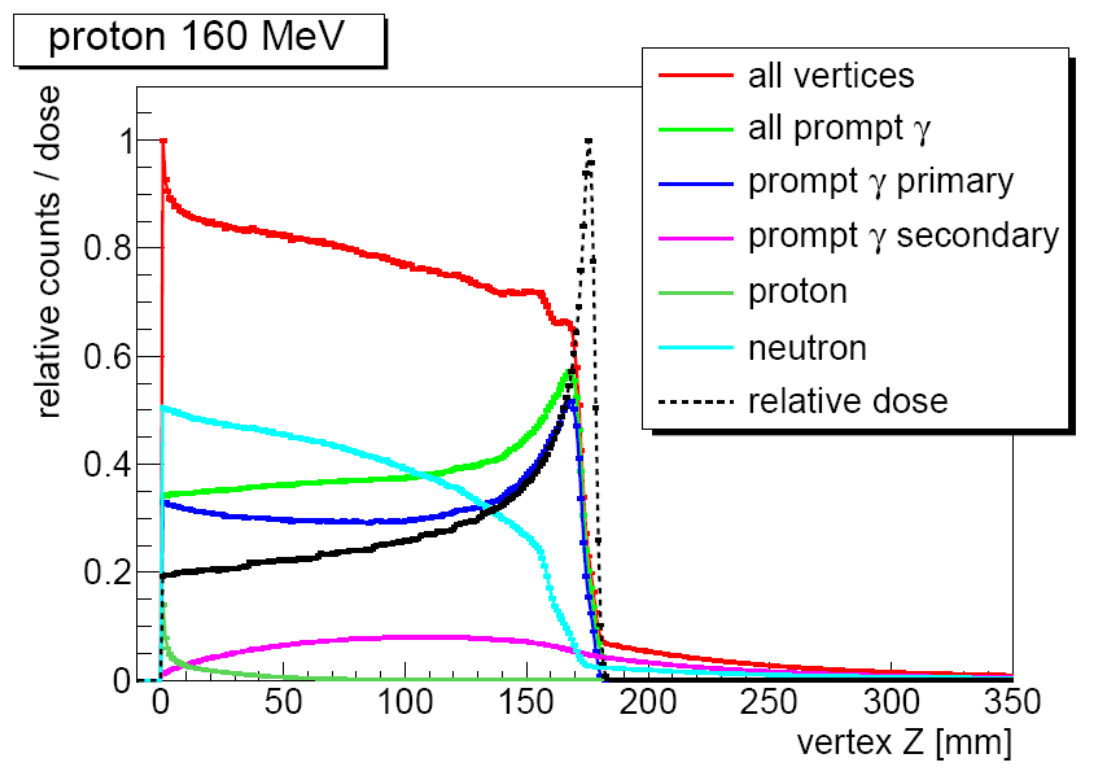

Figure 1. Relative vertex (emission point) distributions of secondary radiation induced by $160 \mathrm{MeV}$ protons incident on a water phantom (15 cm diameter, $40 \mathrm{~cm}$ length). The vertices are scored when the particles emerge from the phantom surface with an energy greater than $1 \mathrm{MeV}$. The longitudinal dose distribution is also presented. Adapted from [25].

A variety of different approaches exploiting PGs are currently being developed, making use of different properties of PG radiation: its spatial, temporal or energy distributions. What they have in common are the conditions and environment in which the developed setups must be operational. Those constraints and challenges are discussed in the next section. Then follows the description of different PG-based methods of range verification, where they have been divided into two groups: imaging and non-imaging techniques (sections 4 and 3). The closing chapter offers a handful of concluding remarks and outlook.

\section{Environment and challenges}

The nuclear collision yield, giving rise to secondary radiation, is typically of the order of a percent per $\mathrm{cm}$ path length for protons. Thus, this represents a fairly high rate at clinical 
beam intensities. In the overall, all accelerators deliver pencil-beam spots $\left(10^{6}-10^{8}\right.$ protons, $10^{4}-10^{6}$ carbon ions) in a fraction of a second (typically 10 to $100 \mathrm{~ms}$ ), which, in turn, yields to $\mathrm{PG}$ emission rates of the order of $10^{7} \mathrm{~s}^{-1}$ in $4 \pi$. A typical PG energy spectrum obtained in an experiment with phantom irradiation is shown in fig. 2. As evoked by Pausch et al. [26],

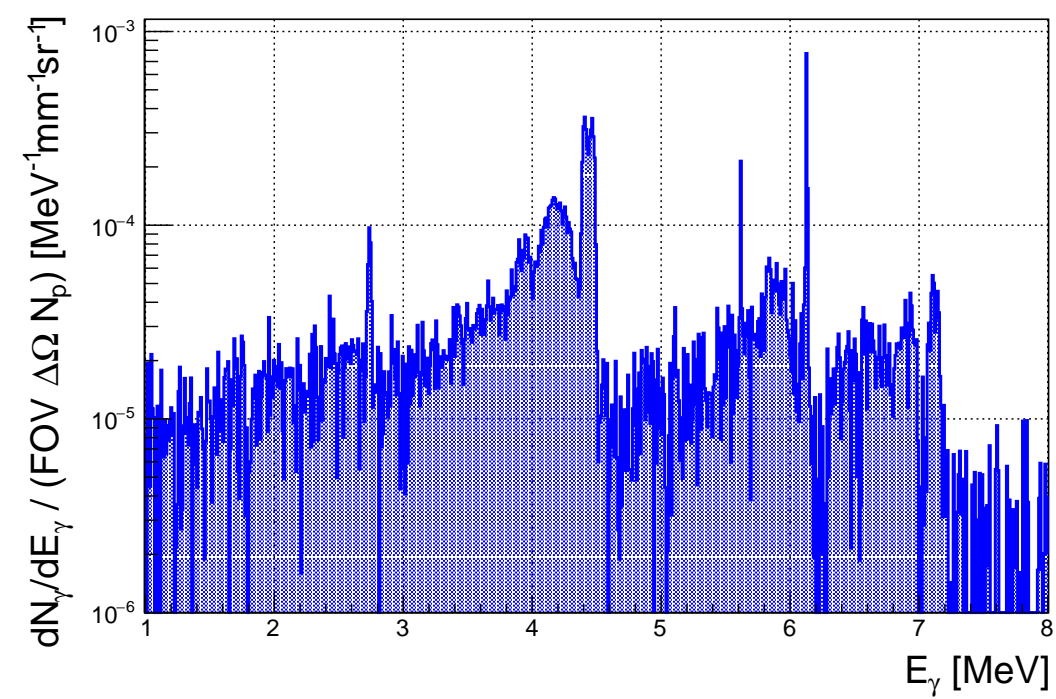

Figure 2. Energy spectrum of PG rays emitted from a 1-mm layer of a PMMA phantom located $2 \mathrm{~mm}$ before the Bragg peak, irradiated with a $70 \mathrm{MeV}$ proton beam. The observed field of view was spatially separated from the degrading (proximal? preceding?) part of the phantom with a $20 \mathrm{~cm}$ air gap to suppress the radiation produced upstream of the depth range of interest. Data recorded with an HPGe detector with an active Compton shield is a part of the set presented in ref. [24]. Yield is normalized by the number of impinging protons, field of view and detector acceptance, corrected for detection efficiency of HPGe, but not for the efficiency of the ACS (increase by $10-25 \%$, depending on energy).

detectors need to cope with both high instantaneous rates, and also with strongly varying count rates within ms. In addition, instantaneous intensities vary strongly from one accelerator type to another. Indeed, the time structure of the delivered beam depends on the type of accelerator, and on the ion species used for treatment. Table 1 illustrates typical characteristics of proton and carbon ion beams at several accelerators. So far, only cyclotrons, synchrotrons and synchrocyclotrons have been exploited for therapy. All machines have a microstructure with periods in the range of nanoseconds, which corresponds to the periodicity of the ion bunches inside the circular accelerator before extraction. On top of this microstructure, there is a macrostructure corresponding to the time separating two cycles of injection/acceleration/extraction, except for cyclotrons for which it is continuous. Therefore, the average PG yields given above may have a much higher instantaneous values, depending on the accelerator duty cycle $(\sim 10 \%$ for cyclotrons and synchrotrons, $\sim 10^{-3}$ for synchro-cyclotrons). This will have an impact for i) time of flight measurement for background rejection and ii) random coincidence rates when a signal corresponds to coincidences with several sub-detectors (Compton cameras).

For ions heavier than protons, on the one side fragmentation rates are higher, since the fragmentation of the projectile itself occurs, but, on the other side, the number of incident projectiles needed to provide a given physical dose is smaller than for protons, due to the $1 / Z^{2}$ dependence of energy loss ( $Z$ being the atomic number), and the lower longitudinal and angular scattering of heavier ions. In addition, the Relative Biological Effectiveness (RBE) leads to a 


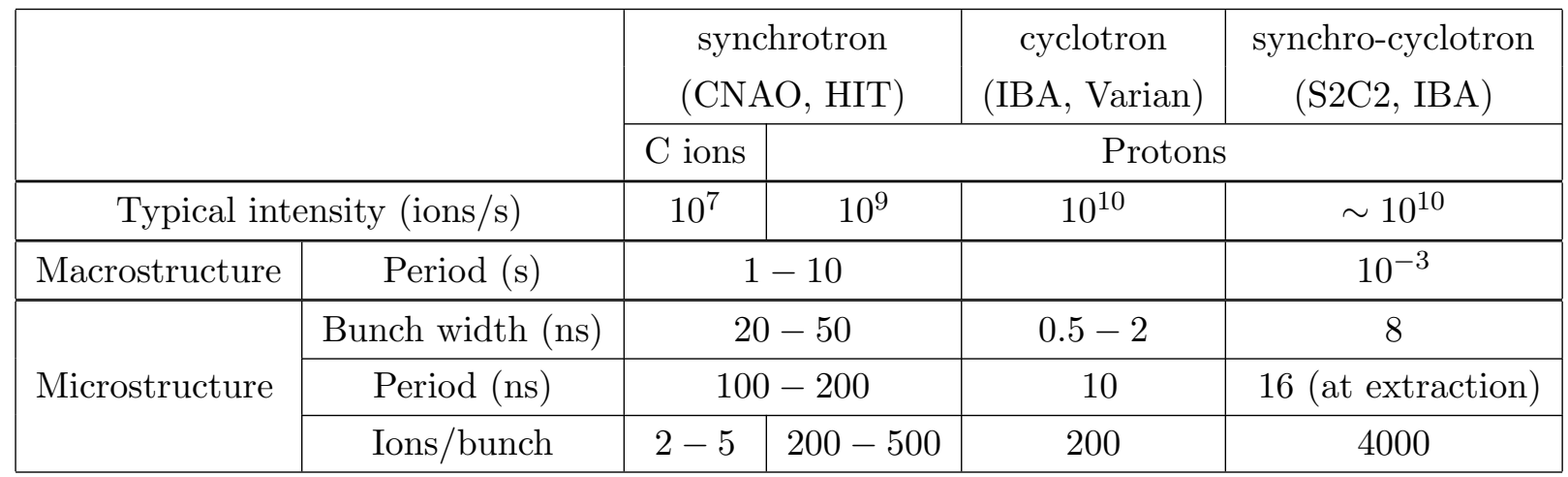

Table 1. Typical time structures of various clinical accelerator types. Note that some other synchrotrons have higher intensities [27]. The numbers were taken from ref. [25].

further reduction of the number of ions necessary to deposit the desired biological dose in the spread-out Bragg peak region with higher- $Z$ ions. Therefore, the number of available PGs for a given beam spot decreases when $Z$ increases: for carbon ions it is typically two orders of magnitude smaller than for protons.

\section{Imaging techniques}

The approaches described in this section aim at range verification by means of the analysis of spatial distribution of $\mathrm{PG}$ vertices, i.e. are based on imaging. That can be performed in one, two or even three dimensions, depending on the complexity of the detection setup and analysis methods.

\subsection{Knife-edge shaped slit-camera (KES)}

A slit camera is probably the most natural approach, an analogue of a pinhole camera, but providing one-dimensional imaging, see fig. 3. Feasibility studies were performed by several groups [28-30]. The worldwide first test of a slit-camera setup in clinical conditions during patient treatment was reported in ref. [31] by the Dresden group in collaboration with OncoRay and IBA. Their camera was mounted on a movable trolley and consisted of a knife-edge shaped tungsten collimator and an array of $4 \mathrm{~mm}$ wide scintillating strips made of LYSO, read out by silicon photomultipliers (SiPMs). The authors focused on the monitoring of inter-fractional range variations rather than absolute range determination and the treatment modality was passively scattered proton therapy. Those variations were determined to be within $\pm 2 \mathrm{~mm}$.

The first clinical trial including prompt-gamma verification during a whole treatment was performed with a second knife-edge prototype from IBA at Philadelphia, using pencil-beam scanning conditions [32]. The analysis of the data acquired during 6 fractions of a brain treatment, performed by comparing measurements to simulations, revealed that the precision in range verification was better than the safety margins applied in the treatment plan.

A collimated system with many knife-edge shaped slits was studied by Ready et al. [33; 34]. In this design the slits formed a $2 \mathrm{D}$ pattern, thereby allowing $2 \mathrm{D}$ imaging. The gamma quanta were detected using a position-sensitive LFS detector. Experimental tests were performed with a $50 \mathrm{MeV}$ proton beam of clinical beam current, showing the precision of relative Bragg peak localization of about $1 \mathrm{~mm}(2 \sigma)$ at the delivery of only $1.8 \times 10^{8}$ protons. A serious limitation, though, was the distance of the collimator front face to the beam axis of only $8 \mathrm{~cm}$. For larger distances the collimator would need to be re-optimized. Measurements with larger proton beam energies are also needed to verify the performance in realistic proton therapy conditions. 


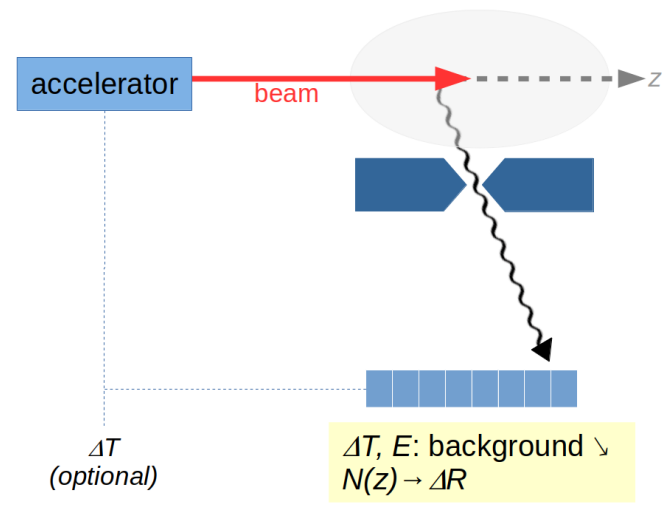

Figure 3. Principle of operation of a slit camera. PG rays are imaged using a single slit onto a position-sensitive detector. Spatial hit distribution allows to conclude about range shifts, while time and energy information may be used to suppress background.

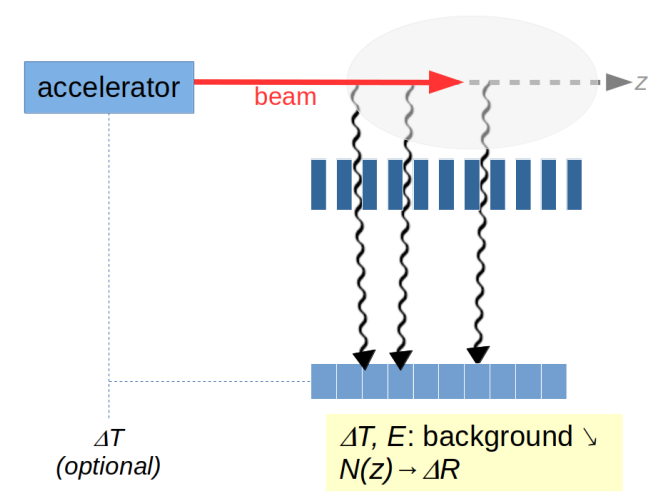

Figure 4. Principle of operation of a multi-parallel slit camera, similar to that of a single slit camera. However, due to a larger field of view, the setup allows to image not only the Bragg-peak region, but also the entrance point, providing full range control.

\subsection{Multi-slit camera (MPS)}

A generalization of a slit-camera concept is a multi-slit camera, which presents the theoretical advantage of a non-restricted field of view. Smeets et al. compared experimentally (using beam energies of 100, 160 and $230 \mathrm{MeV}$ ) two collimated setups: one with their optimized knife-edge shaped collimator (KES), and the other with a multi-slit collimator (MPS) of the same weight [35], the slits parallel to each other. Each collimator was combined with the same detector setup, described in the first paragraph of this section. Due to a smaller field of view, KES provided imaging of the Bragg peak region only, while MPS allowed to image also the beam entrance point. However, in terms of the range retrieval, KES was shown to require only half of the dose MPS needed to obtained the same statistical precision. Thus, the authors concluded KES be the favourable option for further development. However, such conclusions are biased by the constraints put on the MPS camera, which was not independently optimized, as in [36].

A similar collimated setup was built and tested experimentally by Park et al. [37]. The geometry of both the collimator (coarser pattern) and the detector (finer granulation) was somewhat different than in case of [35]. The detector was made of $\mathrm{CsI}(\mathrm{Tl})$ and read out by photodiodes, as more radiation-hard than SiPMs. The authors presented reconstructed gamma depth profiles for different beam energies from the range $95-186 \mathrm{MeV}$, and for each beam energy for four different numbers of delivered protons, between $7.5 \times 10^{7}$ and $7.5 \times 10^{9}$. It turned out possible to locate the distal dose falloff (d90\%), defined as the depth of distal $90 \%$ dose in a depth-dose curve, within about $2-3 \mathrm{~mm}$ of error for the spots which were irradiated with at least $3.8 \times 10^{8}$ protons, regardless of the beam energy. In a typical realistic treatment plan such a dose is delivered only to some of the spots.

\subsection{Gamma-electron vertex imaging (GEVI)}

In the GEVI method, PG rays are not detected directly. Instead, recoil electrons emitted from a beryllium converter as an effect of PG Compton scattering are detected and tracked. The concept was first proposed by a Korean group [38], that recently showed experimental results with test beams [39]. The electron tracking is done with the use of a three-stage hodoscope, shown schematically in fig. 5, consisting of two double-sided silicon strip detectors and a calorimeter 
made of plastic scintillator. The electron tracks are reconstructed based on the hit positions in the silicon detectors. An image is obtained from a back-projection of those tracks onto the imaging plane. Efficient background suppression is achieved by demanding a triple coincidence and imposing cuts on energy depositions in each detector module. The group performed test experiments with an HDPE phantom and $6.24 \times 10^{9}$ delivered protons for seven beam energies between 90 and $180 \mathrm{MeV}$. For the purpose of beam range determination, the obtained twodimensional images were projected onto the beam axis, forming 1D profiles. There, the observed distal falloff at each energy occurred at $94 \%$ of the proton range. According to the authors, the method allows to determine proton range with a precision of $2.7 \mathrm{~mm}$ without the necessity of using Monte-Carlo simulations as reference. One should observe, though, that all results were obtained with the same phantom material. For phantoms with other elemental composition (or in case of a real patient treatment) the shape of the distal falloff may be different. Thus, it remains an open question whether the factor 0.94 is indeed universal. What still needs to be explained is a much lower than expected imaging sensitivity of the system $\left(1.6 \times 10^{-6}\right.$ versus $1.2 \times 10^{-5}$ ), which is probably due to dead time. Nevertheless, the setup, mainly because of its small material budget and simplicity, is an appealing candidate for clinical applications.

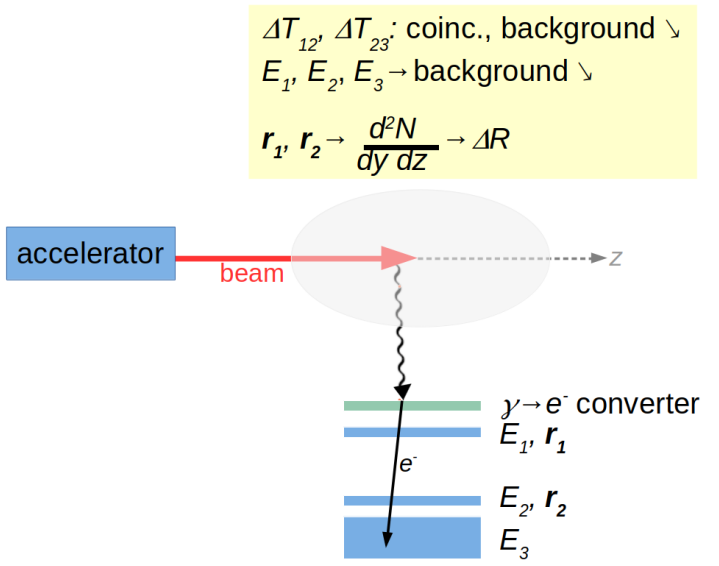

Figure 5. The GEVI method relies on detection if recoil electrons via Compton scattering of $\mathrm{PG}$ in a converter. Triggered by a triple coincidence, the system registers hit positions and energy depositions. Electron tracks are reconstructed and back-projected onto the imaging plane, forming a $2 \mathrm{D}$ image, which is then projected onto the beam axis, delivering information about beam range. Patterns of energy depositions are used to suppress background from other particle species.

\subsection{Compton camera $(C C)$}

A direct reconstruction of a three-dimensional map of $\mathrm{PG}$ activity induced by an irradiation is possible with setups of the Compton camera type (CC). In its classical form, such a setup consists of two modules: a scatterer and an absorber, as depicted in fig. 6. Its operation relies on 
detection of events in which an impinging gamma undergoes Compton scattering in the scatterer and subsequently is fully absorbed in the absorber, preferably via photoeffect. Registration of hit positions as well as associated energy depositions allows to reconstruct a cone of possible directions of the impinging gamma. Additional tracking of the Compton-scattered electron allows to constrain it further to a part of that cone. The image is formed by superposition of the reconstructed Compton cones and though in principle three-dimensional, it is characterized by a rather poor resolution in the direction normal to the detector front face. This, however, can be cured by using a combination of two CCs observing the target from perpendicular directions. One additional difficulty in the case of PG detection is the broad spectrum of photon energies. This requires either the hypothesis of a full energy absorption, or the use of three-stage detection. The PG imaging by means of Compton cameras of various designs was approached by several groups. In the following the main features of the studied CCs are described along with their obtained performance.

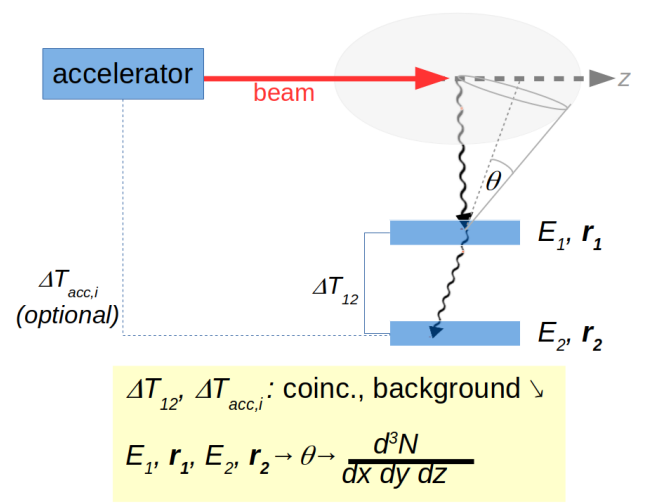

Figure 6. Principle of operation of a Compton camera. Hit positions and energy depositions in the two modules can be translated to a cone of possible directions of impinging PGs. Superposition of such cones delivers a three-dimensional map of PG activity. Timing information is used for background suppression.

- Within the CLaRyS project, the Lyon group studied via simulations a CC with a scatterer made of double-sided silicon strip detectors and an absorber consisting of 100 streaked BGO crystals [40]. A semiconducting scatterer ensured very good position and energy resolution, and a scintillating absorber provided large detection efficiency. A similar setup was built in Munich by Thirolf et al., with an $0.5 \mathrm{~mm}$ thick Si scatterer and a monolithic $\mathrm{LaBr}_{3}$ crystal read out by a multi-anode PMT as an absorber [41]. The image reconstruction procedure took into account also the electron track data. An image resolution of $3.7 \mathrm{~mm}$ was achieved when imaging point-like sources of $1.33 \mathrm{MeV}$ energy. In order to increase the detection efficiency of the scatterer, it was considered to replace the scatterer with a pixelated GAGG detector developed in Japan (see below) [42].

- The Dresden group proposed and characterized a CC of the same type (semiconductor plus scintillator) [43]. For the scatterer, however, instead of the silicon detectors a set of two two cross-strip CZT detectors was used, and the absorber was a single block of LSO, later replaced by a set of three segmented BGO detectors, each of them read out by a set of 4 
classical photomultipliers. The tests performed with a $4.4 \mathrm{MeV}$ gamma source proved the feasibility of imaging at such energies [44]. However, the determined setup efficiency and deduced number of expected registered PG events were by far insufficient for imaging at clinical dose rates. No extension to a clinical size was undertaken.

- The Baltimore group investigated a setup based solely on commercially available, semiconducting CZT detectors [45]. Those detectors have the advantage of excellent energy resolution, but have much worse time resolution and longer signals compared to fast scintillators. Polf et al. performed extensive performance tests of their multi-stage CC. Recently, results of measurements with a small-scale prototype under clinical conditions were reported [46], proving the feasibility of three-dimensional imaging. For that purpose the group developed sophisticated energy reconstruction and event selection methods. The paper reports sensitivity to detect beam range shifts of 2-3 $\mathrm{mm}$, depending on the irradiation scheme.

- An orthogonal design using only scintillating detectors was proposed by the Valencia group. In the approach of Llosá et al., the MACACO Compton telescope consists of monolithic blocks of $\mathrm{LaBr}_{3}$ scintillator read out by arrays of SiPMs [47]. In its first version, the setup consisted of two modules and its capability to image gamma sources of $2-7 \mathrm{MeV}$ energy with 3-5 $\mathrm{mm}$ resolution was shown. Although the group demonstrated also the potential to observe range shifts of $10 \mathrm{~mm}$ for a $150 \mathrm{MeV}$ beam, the available time resolution and detection efficiency still appeared as a problem. After further R\&D addressing those problems, the group reported performance of a modified, three-stage setup featuring an improved detector plane [48].

- Another scintillator-only design was proposed by the Japanese group. Their lightweight and compact (handheld) setup was built of modules consisting of small 'pixel' GAGG crystals of the dimensions $2 \times 2 \times 4 \mathrm{~mm}^{3}$ (scatterer) and of $2 \times 2 \times 2 \mathrm{~mm}^{3}$ (absorber), read out by SiPMs [49]. The initial version of the setup did not have sufficient resolution to deliver conclusive information about possible range shifts in proton therapy. The group introduced modifications, among others reducing all pixels to be cubes of $2 \mathrm{~mm}$ and substituting SiPMs with multi-anode PMTs. In the following tests an attempt to image 4.4 MeV gammas emitted from PMMA phantoms irradiated with a $70 \mathrm{MeV}$ proton beam was undertaken [50]. However, the measurements were performed at the conditions far from clinical - beam current of $3 \mathrm{pA}$ and measurement time of $5 \mathrm{~h}$ allowed to collect vast statistics with a suppressed contribution of random coincidences. Although the capability to use $4.4 \mathrm{MeV}$ PG rays for imaging was demonstrated, no quantitative analysis of range retrieval or feasibility of use in clinical conditions were presented.

- The SiFi-CC project pursued by the Cracow-Aachen collaboration aims at building a Compton camera composed of fibers made of heavy scintillator read out by SiPMs. Laboratory tests and Monte-Carlo simulations indicated that the optimal building blocks for such a setup would be $1 \times 1 \times 100 \mathrm{~mm}^{3}$ LYSO fibers [51]. High granularity of the detectors will allow to reduce the problem of pile-ups occurring at high count rates. The core of the data acquisition system will be custom FPGA-based boards developed for the J-PET project [52], adapted to feature the ADC functionality. Based on the simulations, the expected imaging sensitivity of $0.001 \%$ and the contribution of random coincidences at clinical conditions make imaging of a single beam spot an achievable goal [53]. Currently the group is constructing a small-scale prototype, which will allow one to optimize detector design and performance.

An alternative approach to Compton imaging was proposed by the Dresden group in collaboration with OncoRay [54]. The setup comprising Directional Gamma Radiation Detector (a segmented scintillator) is used in the modality known as Single Plane Compton Imaging, 
taking advantage of the angular distribution of Compton scattering. However, the initial tests with two $2^{\prime \prime} \times 2^{\prime \prime} \mathrm{CeBr}_{3}$ detectors showed that conclusive results useful for range verification require $10^{3}-10^{4}$ times the statistics obtained for a single spot.

In parallel to hardware developments, a lot of effort is put into improving event selection and image reconstruction algorithms. Advanced reconstruction algorithms can handle more complex event topologies, e.g. three-interaction events which allow to determine the energy of the primary gamma [46]. Another approach, reported recently in [55], takes into account the probability of incomplete energy deposition of a primary gamma in the detector. The aspect of computation speed was addressed in [56], where it was shown that a fast line-cone reconstruction technique yields significantly lower precision than an iterative maximum likelihood expectation maximization algorithm. An issue related to the yield of random coincidences between the several detection stages was addressed by several authors [56-58]. A solution for Compton imaging of PG should feature a compromise between the requirement of maximized detection efficiency and reduced background. The tests performed so far indicated that good time resolution allowing to reduce coincidence time and dead time to minimum, high-throughput electronics and data acquisition system and large degree of granularity seem to be key features to build a setup operational at clinical conditions.

\subsection{D imaging with dual $P G$ coincidence}

Panaino et al. make use of coincidence between two consecutive gamma-rays from the deexcitation of ${ }^{16} \mathrm{O}(2.75 \mathrm{MeV}$ followed by $6.13 \mathrm{MeV}$ with 25 ps decay time) to reconstruct in 3D the emission vertex position, by means of a nearly- $4 \pi$ multi-detector of high temporal resolution [59]. This is made possible since the two gamma emissions occur at the same location. In addition, the corresponding excitation cross section by proton impact is maximum at energies close to $13-14 \mathrm{MeV}$, the vertex distribution is maximum very close to the Bragg peak. Although it seems hardly conceivable to install a $4 \pi$ multi-detector with spectroscopic and time resolution capabilities to perform such an imaging in clinical environment, this example, making use of time, spectroscopic and position information, presents an interesting approach of making full use of the setup potential.

\section{Non-imaging techniques}

The second group of methods of beam range verification comprises non-imaging techniques, which take advantage of the correlation between the beam range and the features of temporal or spectral characteristics of PG emission.

\subsection{Prompt-gamma timing (PGT)}

Instead of recording the spatial distributions of PG radiation, Golnik et al. proposed a novel concept of range assessment by prompt-gamma timing (PGT) [60]. The concept is based on the fact, that the transient time of ions in the patient tissue before stopping depends on the proton range. Only during this time PG photons can be produced. Since excited nuclei lifetimes associated to PG emission are shorter than this transient time, the ToF distribution of PG detection relative to the impact of protons is also correlated to the ion range. The concept was somehow approached by Testa et al. by considering ToF distributions of PG emitted from a collimator-restricted field of view, containing the Bragg peak position [61]. In practice, the easiest way to implement PGT is to use a fast detector for PG detection (preferably in backward direction so that flight times of the transient ion and PG are adding), and to synchronize the detection with the accelerator HF signal to provide a stop for ToF measurement, as depicted in fig. 7. However, this necessitates to adapt the time calibration whenever the beam energy changes. Moreover, the beam pulse width is an intrinsic limitation of the method, since the time distribution results from the convolution of individual transient times with the pulse width. 
In particular this is not possible with long lasting beam pulses as delivered by synchrotrons. Alternatively to the cyclotron HF, a signal from a beam pulse monitor can be used for direct ToF measurement. The distribution of time elapsed between the two signals, the so called PGT spectrum, allows to conclude about the proton range by comparing it with the PGT spectrum modelled in the simulations. The simplicity of the setup is an undeniable advantage of this approach.

After initial tests constituting a proof of principle [62], developments towards translation of the method to clinics have been undertaken and reported recently [63]. For this purpose, a series of measurements was performed using a phantom with air cavities and a close-torealistic treatment plan. The detection unit consisted of a $\mathrm{CeBr}_{3}$ crystal coupled to a classical photomultiplier tube (PMT). The scintillator material was chosen for its short decay time and excellent energy resolution. The signals were fed to and processed in a custom FEE unit U100 attached to the PMT, analyzed for a single spot or summed up over the whole iso-energy layer. In the first case there was clearly insufficient statistics, calling for additional detection units. Finally, the PGT spectra for a layer of about $10^{2}$ iso-energy spots were conclusive and provided a precision of 2-3 $\mathrm{mm}$ for 8 detection units. Further developments by the group included the setup of extra detector units, and the increase of their rate capacity, as well as more tests in the clinical conditions.

Marcatili et al. studied the possibility to perform high time-resolution PGT at reduced beam intensity, using a diamond beam trigger working in single-proton counting mode, and fast gamma-ray scintillation detectors $\left(\mathrm{BaF}_{2}\right.$ and $\left.\mathrm{LaBr}_{3}\right)$ [64]. An experimental resolution of 100 ps rms was obtained on the PG-ToF with $65 \mathrm{MeV}$ proton beams using such a system. The simulations undertaken, accounting for such a resolution with the same detection setup, led to a sensitivity to detect an air cavity of $3 \mathrm{~mm}$ located at about $1 \mathrm{~cm}$ from the end of the range with $95 \%$ confidence level with a single beam spot of $10^{8}$ protons. The improvement with respect to the spot-based PGT technique comes from i) the independence on the beam spot duration and ii) the better ToF to proton-position correlation due to the excellent timing resolution.

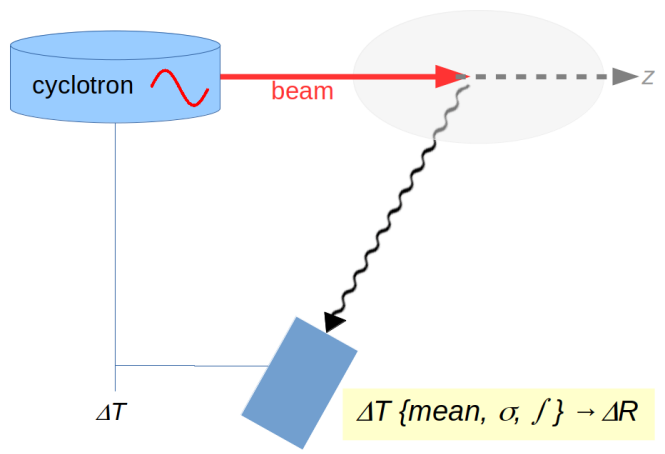

Figure 7. Principle of the PGT and PGPI methods. Both of them rely on recorded distributions of time between beam bunch and registered gamma rays. Analysis of the mean, width and integral of those distributions yields information about beam range. Note that $\Delta T$ can be obtained by means of a beam monitor triggering beam pulses, instead of the cyclotron HF signal. 


\subsection{Prompt-gama peak integrals (PGPI)}

In its original form, PGT method discussed above uses the statistical momenta of the timing distributions, i.e. its mean and width. Krimmer et al. proposed to add to those features also peak integrals of the PGT spectra, while relaxing the constraints on time resolution to a level of 1-2 ns, so as to select only PG emitted inside the patient tissues, and not from the beam nozzle, and to eliminate neutron-induced background. This variation is called Prompt Gamma Peak Integral (PGPI). In an experiment with $\mathrm{LaBr}_{3}$ and $\mathrm{BaF}_{2}$ detectors, a PMMA phantom and a beam of about $65 \mathrm{MeV}$ energy passing through a modulator wheel, the sensitivity of peak integrals to the proton range in the phantom was demonstrated. The used energy range is a lower limit of that used clinically, a favourable one for PG measurements due to lower count rate and neutron background. Thus the authors used Monte-Carlo methods, benchmarked at that energy, to simulate the setup response at higher energies. A precision of about $3 \mathrm{~mm}$ in range verification was obtained for a $10^{8}$-proton pencil beam and a single detector of $25 \mathrm{msr}$ solid angle. In the multiple-detector setup it is possible to additionally detect transverse target misplacements by comparing signals from different detector units.

\subsection{Prompt-gamma spectroscopy (PGS)}

Prompt Gamma Spectroscopy (PGS) is an approach proposed by Verburg et al. [65; 66] from MGH Boston, exploiting spectral characteristics of the PG emission. It requires the registration of PG spectrum with a spectroscopic detector, collimated and focused on a part of the beam path a few millimeters upstream of the Bragg peak. Fig. 8 shows a schematic of such a setup. Cross sections of inelastic proton interactions with the traversed medium are energy dependent. Moreover, depth profiles of gamma emission for different discrete transitions, e.g. from the reactions such as ${ }^{12} \mathrm{C}\left(p, p \gamma_{4.44 \mathrm{MeV}}\right)^{12} \mathrm{C}$ and ${ }^{16} \mathrm{O}\left(p, p \gamma_{6.13 \mathrm{MeV}}\right)^{16} \mathrm{O}$, are different. Therefore the ratios of yields of gammas from various spectral lines can be recalculated to the mean proton energy in the observed slice and, consequently, connected to the residual range. Last but not least, the mentioned ratios allow one to conclude about the elemental composition of the tissues in the observed region. The proof-of-principle experiments were performed using an $\mathrm{LaBr}_{3}$ detector with an active anti-Compton shield (ACS) made of BGO, both with classical PMTs readout. As a collimator a $15 \mathrm{~cm}$ thick lead brick was used. ACS response as well as the hit time information relative to cyclotron timing were used to suppress Compton continuum and neutronincuced components in the spectra, respectively. However, in the first experiments also the small statictics issue appeared and a summation over a whole iso-energy layer had to be performed. To overcome this, a new setup with eight $\mathrm{LaBr}_{3}$ detectors, a tungsten collimator and a dedicated custom high-throughput electronics $\left(10^{7} \mathrm{~s}^{-1}\right)$ was constructed as a clinical prototype [67]. A support frame made it possible to adjust the apparatus to the irradiation direction. Using sophisticated algorithms of background suppression the group achieved a $1.1 \mathrm{~mm}$ precision of beam range verification in measurements with a water phantom and a proton beam of clinically relevant current and dose. As the next steps, commissioning with an anthropomorphic phantom and clinical study with patients are planned.

The application of the PGS method for heavier ion beams was recently presented by Dal Bello et al. [68]. The measurements were performed in HIT Heidelberg using proton, ${ }^{4} \mathrm{He},{ }^{12} \mathrm{C}$ and ${ }^{16} \mathrm{O}$ beams accelerated in a synchrotron, with most effort devoted to study the ${ }^{12} \mathrm{C}$ beams. The setup comprised a $\mathrm{CeBr}_{3}$ detector with a BGO ACS, both equipped with classical PMTs and read out by Flash ADC modules, and a $12.5 \mathrm{~cm}$ thick tungsten collimator. Time reference information was provided by a beam trigger built of scintillating fibers located in front of the target. The group showed that the developed small-scale prototype setup along with the data analysis routines allowed for absolute measurement of the Bragg peak position for synchrotronaccelerated ${ }^{12} \mathrm{C}$ beams at clinically relevant energies and intensities. However, the reported submillimetric precision of range retrieval required delivery of $8 \cdot 10^{9}$ primary ions, which is 


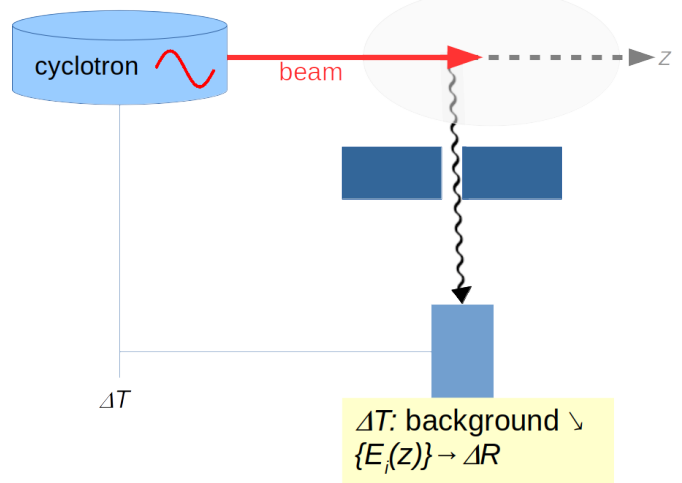

Figure 8. Principle of the PGS method: PG energy is registered by a spectroscopic detector. From yields ratios of PGs originating from different discrete transitions one can deduce the residual range. Physical background is reduced by the ACS and time filtering.

much more than the total in a typical treatment plan. Realistically, range verification at 2 Gy dose with 2-mm precision on a single-spot basis seems unfeasible, and on a plane basis would require an increase of the setup efficiency of 15-40 times, depending on the beam ion species.

\section{Conclusions and outlook}

Various approaches to on-line monitoring of particle therapy have been presented. Some of them have already been successfully tested clinically with patients. The only commissioned setup to date providing a three-dimensional verification of the dose is the PET scanner INSIDE. Up to now, online control with $\mathrm{PG}$ has been restricted to $1 \mathrm{D}$ range verification. It remains a challenge to construct a 3D imaging device exploiting PG radiation which would offer similar performance with a smaller material budget and dimensions.

Some authors suggest that the prospective future developments should focus on making full use of information delivered by a cutting-edge, optimized setup [26]. The 3D imaging with PGs e.g. like that presented in sect. 3.5, combined with PGPI and PGT with excellent timing resolution could serve as an example.

Robust identification of range errors by $\mathrm{PG}$ detection techniques requires appropriate algorithms. Powerful tools of machine learning have been employed interpret the data obtained from simulations and experiment. For instance, using the existing IBA-camera, Khamfongkhruea et al. used training data sets to identify the possible sources of errors (e.g. computed tomography (CT)-based range prediction, patient setup, and anatomical changes between fractions) [69]. Then they built a decision tree in order to classify the error sources. This is a first step using learning approaches, and further developments are needed before clinical implementation, in particular the use of realistic PG acquisition (including noise and reduced statistics). Another attempt of using machine learning methods to convert simulated PG vertex distribution into the deposited dose distribution was presented in ref. [70]. The natural next step should be to apply it to experimental data.

The influence of counting rates has been discussed for various detection modalities. In particular, range verification by means of $\mathrm{PG}$ detection at clinical beam intensities needs to 
cope, on the one side, with high instantaneous counting rates, rapidly varying (beam on and off at every spot and even at each accelerator pulse). On the other side, all verification methods are facing the issue of counting statistics in order to reach the best precision with the shortest irradiation period, possibly a single irradiation spot. This was discussed in the paper by Pausch et al. [26]. Verification methods such as Compton imaging definitely require reduced intensities, at least for a few spots. Dauvergne et al. reviewed the advantages of beam intensity reduction for a few spots at the beginning of a treatment fraction, so that irradiation can be controlled in single ion counting conditions, by means of a fast beam monitor and secondary radiation detection. Obvious advantages are foreseen for Compton imaging (reduction of random coincidences, real time imaging with reduced background using line-cone reconstruction enabling ToF-based filtering with centimeter precision, and improving the precision of this reconstruction method to the level of MLEM), PGT at 100 ps scale or even proton radiography. (cite Dauvergne 2020 not yet referenced, accepted Frontiers). The next decade will surely bring new, exciting developments that will hopefully result in clinical systems for online verification of deposited dose distribution, making particle therapy safer for patients.

\section{Acknowledgments}

AW is grateful to the Polish National Science Centre for supporting her work on prompt-gamma imaging with the SONATA BIS grant no. 2017/26/E/ST2/00618. DD is supported by the Laboratory of Excellence LabEx PRIMES (ANR-11-LABX-0063) and the ITMO-Cancer. Both authors would like to thank warmly Etienne Testa, Jean Michel Létang, xx and yy for their help by reading this manuscript.

\section{References}

[1] Wrońska A 2020 Journal of Physics: Conference Series 1561012021 URL https:// iopscience.iop.org/article/10.1088/1742-6596/1561/1/012021/pdf

[2] Wilson R R 1946 Radiology 47 487-91

[3] Linz U (ed) 2012 Ion Beam Therapy (Heidelberg Dordrecht London New York: Springer)

[4] PTCOG web page https://www.ptcog.ch/index.php/facilities-in-operation

[5] Paganetti H 2012 Physics in Medicine and Biology 57 R99-R117

[6] Knopf A C and Lomax A 2013 Physics in Medicine and Biology 58 R131-160 ISSN 13616560

[7] Kraan A C 2015 Frontiers in Oncology 5 ISSN 2234-943X URL http://www.ncbi.nlm. nih.gov/pmc/articles/PMC4493660/

[8] Parodi K 2019 The British Journal of Radiology 9320190787 ISSN 0007-1285 publisher: The British Institute of Radiology URL https://www.birpublications.org/doi/abs/ 10.1259/bjr. 20190787

[9] NuPECC report 2014: Nuclear Physics for Medicine http://www.nupecc.org/pub/ npmed2014.pdf

[10] Parodi K and Assmann W 2015 Modern Physics Letters A 301540025

[11] Lehrack S, Assmann W, Bender M, Severin D, Trautmann C, Schreiber J and Parodi K 2020 Nuclear Instruments and Methods in Physics Research, Section A: Accelerators, Spectrometers, Detectors and Associated Equipment 950162935 ISSN 01689002 (Preprint 1903.12170)

[12] Traini G et al 2019 Physica Medica 65 84-93

[13] Yamaguchi M, Nagao Y, Ando K, Yamamoto S, Toshito T, Kataoka J and Kawachi N 2016 Nuclear Instruments and Methods in Physics Research Section A: Accelerators, 
Spectrometers, Detectors and Associated Equipment 833 199-207 ISSN 0168-9002 00000 URL http://www.sciencedirect.com/science/article/pii/S0168900216307756

[14] Marafini M, Gasparini L, Mirabelli R, Pinci D, Patera V, Sciubba A, Spiriti E, Stoppa D, Traini G and Sarti A 2017 Physics in Medicine and Biology 62 3299-312

[15] Enghardt W, Crespo P, Fiedler F, Hinz R, Parodi K, Pawelke J and Pönisch F 2004 Nuclear Instruments and Methods in Physics Research Section A 525 284-288 ISSN 0168-9002 URL http://www.sciencedirect.com/science/article/pii/S0168900204004218

[16] Parodi K 2015 Medical Physics 42 7153-68

[17] Dendooven P, Buitenhuis H J, Diblen F, Heeres P N, Biegun A K, Fiedler F, Van Goethem M J, Van Der Graaf E R and Brandenburg S 2015 Physics in Medicine and Biology 60 8923-8947 ISSN 13616560 URL https://doi.org/10.1088/1361-6560/ab23d7

[18] Ozoemelam I S, van der Graaf E R, van Goethem M J, Kapusta M, Zhang N, Brandenburg S and Dendooven P 2020 Physics in Medicine E Biology ISSN 0031-9155

[19] Bisogni M G et al 2016 Journal of Medical Imaging 4011005

[20] Ferrero V et al 2018 Scientific Reports 84100

[21] Yamaya T 2017 Journal of Physics: Conference Series 777012023 ISSN 1742-6596 publisher: IOP Publishing URL https://doi.org/10.1088\%2F1742-6596\%2F $777 \% 2 \mathrm{~F} 1 \%$ $2 \mathrm{~F} 012023$

[22] Min C H, Kim C H, Youn M Y and Kim J W 2006 Applied Physics Letters 89183517

[23] Pinto M et al 2015 Physics in Medicine and Biology 60 565-94

[24] Kelleter L et al 2017 Physica Medica 34 7-17

[25] Krimmer J, Dauvergne D, Létang J M and Testa 018 Nuclear Instruments and Methods in Physics Research Section A: Accelerators, Spectrometers, Detectors and Associated Equipment 878 58-73 ISSN 0168-9002 URL http://www.sciencedirect.com/science/ article/pii/S0168900217308380

[26] Pausch G, Berthold J, Enghardt W, Römer K, Straessner A, Wagner A, Werner T and Kögler T 2020 Nuclear Instruments and Methods in Physics Research Section A: Accelerators, Spectrometers, Detectors and Associated Equipment 954161227 ISSN 01689002 URL http://www.sciencedirect.com/science/article/pii/S0168900218312063

[27] Kanazawa M, Endo M, Himukai T, Kitamura M, Mizota M, Nakagawara A, Sato H, Shioyama Y, Totoki T and Tsunashima Y 2017 Scanning Irradiation System at SAGAHIMAT (JACOW, Geneva, Switzerland) pp 4698-4700 ISBN 978-3-95450-182-3 URL http://accelconf .web.cern.ch/ipac2017/doi/JACoW-IPAC2017-THPVA101.html

[28] Smeets J et al 2012 Physics in Medicine and Biology 57 3371-405

[29] Perali I et al 2014 Physics in Medicine and Biology 59 5849-71

[30] Cambraia Lopes P et al 2015 Physics in Medicine and Biology 60 6063-85

[31] Richter C et al 2016 Radiotherapy and Oncology 118 232-7

[32] Xie Y, Bentefour H, Janssens G, Smeets J, Stappen F V, Hotoiu L, Yin L, Dolney D, Avery S, O'Grady F, Prieels D, McDonough J, Solberg T D, Lustig R, Lin A and Teo B K K 2017 International Journal of Radiation Oncology • Biology • Physics 0 ISSN 0360-3016 URL http://www.redjournal .org/article/S0360-3016\%2817\%2930848-9/abstract

[33] Ready J, Negut V, Mihailescu L and Vetter K 2016 Medical Physics 433717

[34] Ready J F 2016 Development of a multi-knife-edge slit collimator for prompt gamma ray imaging during proton beam cancer therapy Phd University of California, Berkeley URL http://adsabs.harvard.edu/abs/2016PhDT .......151R 
[35] Smeets J, Roellinghoff F, Janssens G, Perali I, Celani A, Fiorini C, Freud N, Testa E and Prieels D 2016 Frontiers in Oncology 6156

[36] Pinto M, Dauvergne D, Freud N, Krimmer J, Letang J M, Ray C, Roellinghoff F and Testa E 2014 Physics in Medicine and Biology 59 7653-7674 ISSN 1361-6560

[37] Park J H, Kim S H, Ku Y, Kim C H, Lee H R, Jeong J H, Lee S B and Shin D H 2019 Nuclear Engineering and Technology 51 1406-16

[38] Kim C H, Park J H, Seo H and Lee H R 2012 Medical Physics 39 1001-5

[39] Kim C H, Lee H R, Kim S H, Park J H, Cho S and Jung W G 2018 Applied Physics Letters $113114101-5$

[40] Krimmer J et al 2015 Nuclear Instruments and Methods in Physics Research Section A: Accelerators, Spectrometers, Detectors and Associated Equipment 787 98-101

[41] Aldawood S et al 2017 Radiation Physics and Chemistry 140 190-7

[42] Liprandi S et al 2017 Characterization of a Compton camera setup with monolithic $\mathrm{LaBr} 3(\mathrm{Ce})$ absorber and segmented GAGG scatter detectors Proceedings of 2017 IEEE Nuclear Science Symposium and Medical Imaging Conference (NSS/MIC) (IEEE) ISBN 978-1-5386-2282-7

[43] Hueso-González F et al 2014 Journal of Instrumentation 9 P05002

[44] Golnik C et al 2016 Journal of Instrumentation 11 P06009

[45] Polf J C, Avery S, Mackin D S and Beddar S 2015 Physics in Medicine and Biology 60 7085-99

[46] Draeger E, Mackin D, Peterson S, Chen H, Avery S, Beddar S and Polf J C 2018 Physics in Medicine and Biology 63035019

[47] Llosá G et al 2013 Nuclear Instruments and Methods in Physics Research, Section A: Accelerators, Spectrometers, Detectors and Associated Equipment $\mathbf{7 1 8}$ 130-3

[48] Barrio J, Etxebeste A, Granado L, Muñoz E, Oliver J F, Ros A, Roser J, Solaz C and Llosá G 2018 Nuclear Instruments and Methods in Physics Research, Section A: Accelerators, Spectrometers, Detectors and Associated Equipment 912 48-52

[49] Taya T, Kataoka J, Kishimoto A, Iwamoto Y, Koide A, Nishio T, Kabuki S and Inaniwa T 2016 Nuclear Instruments and Methods in Physics Research Section A: Accelerators, Spectrometers, Detectors and Associated Equipment 831 355-61

[50] Koide A et al 2018 Scientific Reports 88116

[51] Wrońska A, Hetzel R, Kasper J, Lalik R, Magiera A, Rusiecka K and Stahl A 2020 Acta Physica Polonica B $\mathbf{5 1}$ 17-25

[52] Niedźwiecki S et al 2017 Acta Physica Polonica B 48 1567-76

[53] Kasper J, Rusiecka K, Hetzel R, Kazemi Kozani M, Lalik R, Magiera A, Stahl A and Wrońska A 2020 Physica Medica 76 317-325 ISSN 11201797 URL https://linkinghub. elsevier.com/retrieve/pii/S1120179720301733

[54] Pausch G, Golnik C, Schulz A and Enghardt W 2017 A novel scheme of Compton imaging for nuclear medicine 2016 IEEE Nuclear Science Symposium, Medical Imaging Conference and Room-Temperature Semiconductor Detector Workshop, NSS/MIC/RTSD 2016 vol 2017January (Institute of Electrical and Electronics Engineers Inc.) ISBN 9781509016426

[55] Muñoz E, Barrientos L, Bernabéu J, Borja-Lloret M, Llosá G, Ros A, Roser J and Oliver J F 2020 Physics in Medicine and Biology 65025011 ISSN 13616560 URL https: //doi.org/10.1088/1361-6560/ab58ad 
[56] Fontana M, Testa E, Ley J L, Dauvergne D, Freud N, Krimmer J, Letang J M, Maxim V, Richard M H and Rinaldi I 2020 IEEE Transactions on Radiation and Plasma Medical Sciences 4 218-232

[57] Ortega P G, Torres-Espallardo I, Böhlen T T, Cerutti F, Chin M P W, Ferrari A, Gillam J E, Lacasta C, Llosá G, Oliver J, Rafecas M, Sala P R and Solevi P 2013 Noise evaluation of prompt-gamma technique for proton-therapy range verification using a Compton Camera 2013 IEEE Nuclear Science Symposium and Medical Imaging Conference (2013 NSS/MIC) pp 1-7 iSSN: 1082-3654

[58] Rohling H, Priegnitz M, Schoene S, Schumann A, Enghardt W, Hueso-González F, Pausch G and Fiedler F 2017 Physics in Medicine and Biology 622795 ISSN 0031-9155 URL http://stacks.iop.org/0031-9155/62/i=7/a=2795

[59] Panaino C M V, Mackay R I, Kirkby K J and Taylor M J 2019 Scientific Reports 918820 ISSN 2045-2322 URL https://doi.org/10.1038/s41598-019-55349-7

[60] Golnik C et al 2014 Physics in Medicine and Biology 59 5399-422

[61] Testa M, Min C H, Verburg J M, Schümann J, Lu H M and Paganetti H 2014 Physics in Medicine and Biology $\mathbf{5 9}$ 4181-95

[62] Hueso-González F et al 2015 Physics in Medicine and Biology 60 6247-72

[63] Werner T et al 2019 Physics in Medicine and Biology 64105023

[64] Marcatili S, Collot J, Curtoni S, Dauvergne D, Hostachy J Y, Koumeir C, Létang J M, Livingstone J, Metivier V, Gallin-Martel L, Gallin-Martel M L, Muraz J F, Servagent N, Testa E and Yamouni M 2020 Physics in Medicine 83 Biology ISSN 0031-9155 URL http://iopscience.iop.org/10.1088/1361-6560/ab7a6c

[65] Verburg J M, Riley K, Bortfeld T and Seco J 2013 Physics in Medicine and Biology 58 L37-L49

[66] Verburg J M and Seco J 2014 Physics in Medicine and Biology 59 7089-106

[67] Hueso-González F, Rabe M, Ruggieri T, Bortfeld T and Verburg J M 2018 Physics in Medicine and Biology 63185019

[68] Dal Bello R, Martins P M, Brons S, Hermann G, Kihm T, Seimetz M and Seco J 2020 Phys. Med. Biol 6595010 URL https://doi.org/10.1088/1361-6560/ab7973

[69] Khamfongkhruea C, Berthold J, Janssens G, Petzoldt J, Smeets J, Pausch G and Richter C Medical Physics n/a ISSN 2473-4209 eprint: https://onlinelibrary.wiley.com/doi/pdf/10.1002/mp.14393 URL http://aapm. onlinelibrary.wiley.com/doi/abs/10.1002/mp.14393

[70] Liu C C and Huang H M 2020 Physica Medica 69 110-119 ISSN 1724191X 